\title{
Hydrothermal treatment of sunflower seeds in grain washing machines with spiral screw devices
}

\author{
Munir Gafin* \\ Technological Institute - branch of the Federal State Budgetary Educational Institution of Higher \\ Education "Ulyanovsk State Agrarian University named after P.A.Stolypin", Dimitrovgrad, \\ Ulyanovsk region, Russian Federation
}

\begin{abstract}
The presented article examines the relevance of the process of preparing sunflower seeds before roasting for the production of snacks by washing as one of the ways to increase the efficiency of this type of production. The article also gives a brief overview of the advantages of washing and the disadvantages of this method of preparing seeds for roasting, and highlights possible ways of leveling the negative consequences of moistening-washing.
\end{abstract}

Roasted sunflower seeds are a very popular product in the CIS countries, incl. in the Russian Federation. Many people like to buy it: not only young people, but also older people. The demand for this product in the snack category is stable. If we compare roasted sunflower seeds with other products from the snack category (croutons, chips, etc.), we can notice that they compare favorably with others snacks because their target audience of their consumers is the widest one.

The popularity of sunflower seeds as a food product can be explained by the following factors:

- $\quad$ no age restrictions: they are allowed to be eaten by children, adults and the elderly;

- $\quad$ no restrictions on the place of use;

- the absence of a negative effect on the human body provided that consumption of this product is moderate;

- $\quad$ seeds contain a lot of substances useful for humans, such as vitamins of groups A, $\mathrm{B}, \mathrm{D}$ and $\mathrm{E}$, zinc, magnesium, and unsaturated fatty acids;

- due to the content of useful substances in the seeds, the eating of this product might help to reduce the level of cholesterol in the blood, to accelerate the regeneration of the skin, to improve the condition of the hair, and also has a calming effect on the human nervous system, which makes it easier, for example, to cope with the rejection of smoking.

In connection with the above, the production of roasted sunflower seeds requires the development of new less energy-intensive and less metal-intensive machines and tools

\footnotetext{
* Corresponding author: mgafin@yandex.ru
} 
available to the middle circle of manufacturers, which would reduce labor, material and energy costs for the production of high quality roasted sunflower seeds.

One of these ways to optimize the production process of the finished product is the technology of mixing seeds with water and centrifuging the seeds in order to remove external moisture from the sunflower seeds using rotating spiral-screw transporting devices.

Improving the technological process of loading seeds into a container, moistening seeds, unloading seeds, removing contaminated water with impurities, removing external moisture from sunflower seeds by centrifugation and supplying seeds to bunkers for roasting by developing a new washing device based on rotating spiral-screw transporting working bodies is an urgent and critical task. The solution to this problem is of great importance on the way of mechanizing labor-intensive processes and reducing costs in the preparation of sunflower seeds, obtaining products of improved quality.

The schematic diagram and design of the device for washing and centrifuging sunflower seeds developed by us are shown in Figure 1 below.

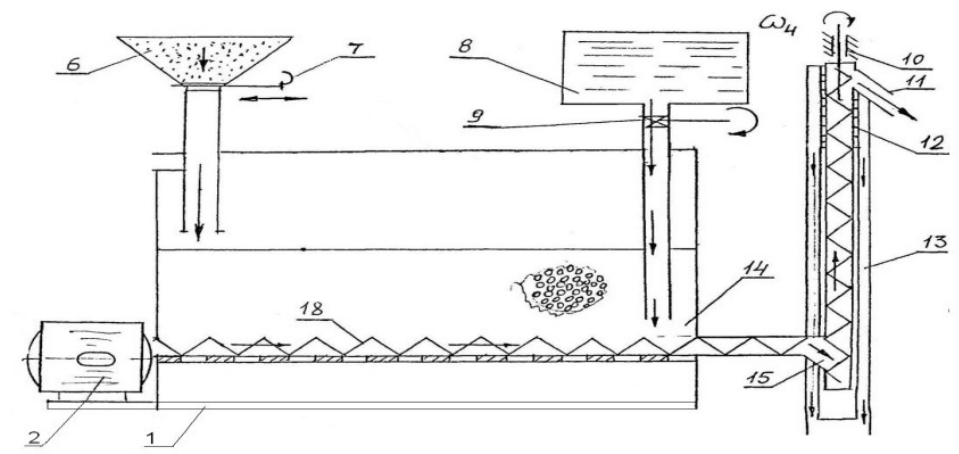

Fig. 1. Scheme of the washing device (side view):1 - frame; 2 - engine; 3, 4, 5, 10 - bearing devices; 6 - seeds; 7 - damper; 8 - water; 9 - crane; 11 - tray; 12 - perforated casing; 13 - fence; 14 - washing space; 15 - washed sunflower seeds.

The working process of the washing device is carried out as follows: dry seeds 6 through the gate 7 enters the chamber 14 and by the spring 18 through the tray 15 enters the centrifuge, rises upward, freed from external moisture, and through the tray 11 enters the feed line; contaminated water with heavy impurities is discharged by a rotating spiral 19 into the sewer; light impurities floating above the water are swept away by spirals 16 partially with water into the sewer (to prevent the sagging of the spirals inside the spiral, cores are stretched); water from the container 8 is fed into the washing space 14 in countercurrent to the seeds 18; external moisture from the seeds is discharged through the perforated casing 12 and is discharged along the fence 13;contaminated water from the space 14 enters the space 20 through the sieve 17.

The main properties of certain seeds and mass are the geometric dimensions of the seeds, their density, the bulk density of the seed layer (nature), the rate of soaring, the mass of 1000 seeds, the angle of repose, heat capacity, and thermal conductivity. These characteristics are divided into three groups: physical, thermophysical and mass transfer[1,2].

All these characteristics in most cases change in the process of moistening and dehydrating seeds. Physical changes in seeds during moistening are externally manifested in a change in the geometric dimensions and density of sunflower seeds. Washing of seeds 
is always accompanied by compression (contraction), since the increase in volume $\Delta V_{3}$ does not always correspond to the volume of absorbed water $\Delta V_{3}$. Then contraction is determined by the ratio:

$$
C_{k}=\frac{\Delta V_{3}-\Delta V_{B}}{G}
$$

where $G$ - seed mass.

Moistening processes occur in the seed layer, which is formed from individual seeds, the packing density of which determines the wellness of the layer, i.e. the ratio of the volume of free spaces, representing air channels, to the total volume of sunflower seeds. The movement of working agents (water, steam and air) passes through these channels.

The speed and features of the interaction of working agents with seeds depend on the aerodynamic characteristics of these channels: shape, cross-section, etc.[3]. The degree of difference between the shape of the seeds and the shape of the ball is estimated by the coefficient of sphericity, which is the ratio of the surface area of the ball, equal in volume to the seed, to its actual surface area. Some of scientists proposed a formula for determining the volume of the seed[4]:

$$
V=k a b l,
$$

where $k$ is the seed shape factor $(k=0,4 \ldots 0,55)$;

$a, b, l$ - linear dimensions.

The surface area of the weevil is determined by calculation:

$$
F=4 \pi R(1+3 R)
$$

where

$$
R=\frac{5 a+6 b}{60} .
$$

The $V / F$ ratio is considered to be the determining or characteristic size. According to the literature, the volume of caryopses of various crops varies within the limits $V_{3}=(12 \ldots 50)$ $\mathrm{mm}^{3}$, the area of the outer surface of the caryopsis is $F=(30 \ldots 80) \mathrm{mm}^{2}$. Wheat sphericity $(0.70 \ldots 0.80)$; barley -0.80 ; oats -0.72 ; rice -0.84 ; sunflower -0.85 . According to other data, the surface area of the seeds layer is determined by the formula:

$$
F=\frac{6 G}{\rho d_{\ni}}
$$

where $G$ is the mass of seeds in the weighed portion (sample);

$\rho, d_{\ni}$ - density and equivalent diameter of seeds.

Or in another expression:

$$
F=\pi d_{\ni}^{2} \phi N
$$

where $N$ is the number of seeds in the sample;

$\varphi$ is a form factor, which for main crops varies within 1.5 ... 2.0.

These ratios make it possible to determine the contact surface of the phases $F_{0}$, the aerodynamic (hydraulic) resistance of the sunflower seed layer, and other characteristics.

When the seeds are moistened and dehydrated, the geometrical dimensions of the caryopses, their density, and other characteristics change. As a result of changes in moisture content, the volume of caryopses increases significantly by (25 ... 30) \%, the area of the outer surface by $(15 \ldots 20) \%$, the equivalent diameter - by (5 ... 10) \%, density - by (10 ... $15) \%$. Changes in density are considered as loosening of the grain structure. 
Of all the characteristics of seeds during moistening, the coefficient of moisture diffusion $a_{\mathrm{m}}\left(\mathrm{m}^{2} / \mathrm{s}\right)$ and the coefficient of thermal and moisture conductivity $\delta$, $(\% / \mathrm{K})$, which characterize the relative thermal transfer of moisture in the form of liquid and vapor under non-isothermal conditions and depend on the moisture content, are important. The coefficient $\delta$ determines the value of the moisture drop in the weevil at a temperature drop of $1^{\circ} \mathrm{C}$.

Various sources indicate that moisture transfer in the seed layer is observed even at an initial moisture content of $4.6 \%$. With a further increase in moisture content, the coefficient of thermal and moisture conductivity increases and in the range (15 ... 17) \% has a maximum. In absolute terms, the coefficient $\delta$ does not exceed $0.4 \% /{ }^{\circ} \mathrm{C}$. Considering the fact that when washing the seeds, the temperature gradient exists for no more than one hour (usually $0.25 \ldots 0.5 \mathrm{~h}$ ), it can be assumed that the effect of the thermal conductivity coefficient on the internal moisture transfer in the seeds layer is insignificant. Moisture diffusion coefficient $a_{\mathrm{m}}$ reflects the nature of moisture transfer and varies with temperature and moisture content. Its average values are $a_{\mathrm{m}}=2,0 \cdot 10^{-11} \mathrm{~m}^{2} / \mathrm{s}$.

Generalized characteristics of moisture transfer consider the numbers of Lykov, Fourier, Kirpichev, Bio, Prandtl, Kosovich, phase transformation, which for grain vary within the following limits: $\quad L u=\frac{a_{m}}{a}=(0,5 \ldots 1,0) \cdot 10^{-4} ; \quad F O=\frac{a \tau}{l^{2}}=0,03 \ldots 0,1 ; \quad K i=\frac{q_{m} l}{a_{m} v_{0} \rho_{0}}$ $=0 . .2,0 ; \quad B i=\frac{\alpha l_{c m}}{\lambda_{c m}} \approx 2,5 ; \quad \operatorname{Pr}=\frac{c \mu}{\lambda}=3,5 \ldots 9,5 \quad\left(\right.$ from $\quad 50 \quad$ to $\left.10 \quad{ }^{\circ} \mathrm{C}\right)$; $K o=\frac{r \Delta u}{c \Delta \theta}=20 \ldots 8$.

If the values of the mass transfer Fourier number $\left(F O_{\mathrm{m}}\right)$, depending on the moisture content, does not exceed 0.1 , then the process of internal moisture transfer in seeds lies entirely in the nonstationary region. If the value of the Lykov number, depending on the moisture content, is $L u=(0,5 \ldots 1,0) \times 10^{-4}$, then the intensity of the development of the temperature field in the caryopsis is $10^{4}$ times higher than the intensity of the development of the field of moisture content. This means that, in practice, thermal and moisture conductivity does not play a significant role in internal moisture transfer.

The dependence of the $K i_{\mathrm{m}}$ number on moisture in the process of sunflower seed dehydration has a maximum. This means that at first the resistance to internal moisture transfer increases, and after some time it decreases. Presumably, an increase in the value of the $K i_{\mathrm{m}}$ criterion can be associated with a deepening of the zone of moisture evaporation, and a decrease - with irreversible changes in the structure of seeds, in particular, with the formation of microcracks in its endosperm. The value of the $K i_{\mathrm{m}}$ criterion decreases with increasing temperature. The transfer of heat and moisture in the seedbed and the single seed is characteristic. This is due to the complex shape, the anatomical structure of sunflower seeds, the uneven distribution of chemical substances along its cross section, as well as the physiological needs of the seeds as a living organism.

The processes of heat and moisture transfer in hydrophilic materials are mutually related. For sunflower seeds, this is expressed, first of all, in the fact that the diffusion coefficient of moisture largely depends on temperature. An increase or decrease in it causes a noticeable change in the intensity of moisture transfer. At the same time, the development of the fields of moisture content and temperature in a single seed occurs independently, and the second is ahead of the first in speed $\left(10^{3} \ldots 10^{4}\right)$ times, which follows from the value of the Lykov criterion. 


\section{References}

1. Artemiev V,Voronina V and Gafin M2008 Transportation of semi-liquid materials by troughs(Ulyanovsk: UGSKhA Press) p 30

2. Gafin M 2018 The efficiency of grain washing in grain washing machines with spiral screw devices depending on the temperature and water hardness Achievement of technology and technologies in the agroindustrial complex: materials of the International Scientific and Practical Conf. dedicated to the memory of V Artemyev (Ulyanovsk: Ulyanovsk State Agricultural University Press) pp 54-60

3. Gafin M 2019Speed of grain movement in grain washing machines based on rotating springsRural mechanic5p 22-23

4. Ginzburg A 1984Thermophysical properties of grain, flour and groats(Moscow: Kolos) p 200 